\title{
Thyroid-stimulating autoantibodies in Graves disease preferentially recognize the free A subunit, not the thyrotropin holoreceptor
}

\author{
Gregorio D. Chazenbalk, ${ }^{1}$ Pavel Pichurin, ${ }^{1}$ Chun-Rong Chen, ${ }^{1}$ Francesco Latrofa, ${ }^{1}$ \\ Alan P. Johnstone, ${ }^{2}$ Sandra M. McLachlan, ${ }^{1}$ and Basil Rapoport ${ }^{1}$ \\ ${ }^{1}$ Autoimmune Disease Unit, Cedars-Sinai Research Institute and School of Medicine, University of California, \\ Los Angeles, Los Angeles, California, USA \\ ${ }^{2}$ Department of Biochemistry and Immunology, St. George's Hospital Medical School, London, United Kingdom
}

Graves disease is directly caused by thyroid-stimulating autoantibodies (TSAb's) that activate the thyrotropin receptor (TSHR). We observed upon flow cytometry using intact cells that a mouse mAb (3BD10) recognized the TSHR ectodomain with a glycosidylphosphatidylinositol (ECD-GPI) anchor approximately tenfold better than the same ectodomain on the wild-type TSHR, despite the far higher level of expression of the latter. The 3BD10 epitope contains the $\mathrm{N}$-terminal cysteine cluster critical for TSAb action. Consequently, we hypothesized and confirmed that TSAb (but not thyrotropin-blocking autoantibodies [TBAb's]) also poorly recognize the wild-type TSHR relative to the ECD-GPI. Despite poor recognition by TSAb of the holoreceptor, soluble TSHR A subunits (known to be shed from surface TSHR) fully neutralized autoantibody-binding activity. These data indicate that the epitope(s) for TSAb's, but not for TBAb's, are partially sterically hindered on the holoreceptor by the plasma membrane, the serpentine region of the TSHR, or by TSHR dimerization. However, the TSAb epitope on the soluble A subunit is freely accessible. This observation, as well as other evidence, supports the concept that A subunit shedding either initiates or amplifies the autoimmune response to the TSHR, thereby causing Graves disease in genetically susceptible individuals.

J. Clin. Invest. 110:209-217 (2002). doi:10.1172/JCI200215745.

\section{Introduction}

Graves disease is a common organ-specific autoimmune disease with a unique feature. Rather than autoimmunity causing organ damage, autoantibodies activate the thyrotropin receptor (TSHR) and increase target organ activity, leading to thyroid hyperplasia, increased thyroid hormone secretion, and clinical thyrotoxicosis (reviewed in ref. 1). Besides being the direct cause of a prototypic autoimmune disease, TSHR autoantibodies and their antigen have a number of intriguing features. The autoantibodies are frequently oligoclonal, as evidenced by restriction in many patients to either lambda or kappa light chains (2-4) and limitation to the IgG1 subclass (5). Consistent with these features, thyroidstimulating autoantibodies (TSAb's) are present at very low concentrations in patients' serum $(6,7)$, typically in the nanogram per milliliter range $(8,9)$, two to three

Received for publication April 19, 2002, and accepted in revised form May 28, 2002.

Address correspondence to: Basil Rapoport, Cedars-Sinai Medical Center, 8700 Beverly Boulevard, Suite B-131, Los Angeles, California 90048, USA. Phone: (310) 423-0555; Fax: (310) 423-0221; E-mail: rapoportb@cshs.org.

Conflict of interest: No conflict of interest has been declared. Nonstandard abbreviations used: thyrotropin receptor (TSHR); thyroid-stimulating autoantibody (TSAb); thyrotropin-blocking autoantibody (TBAb); Chinese hamster ovary (CHO); ectodomain-glycosylphosphatidylinositol (ECD-GPI) orders of magnitude lower than polyclonal autoantibodies to thyroid peroxidase, another cell surface thyroid-specific autoantigen common to both Graves disease and Hashimoto thyroiditis. Very rarely, autoantibodies bind to, but do not activate, the TSHR, thereby blocking the action of TSH and causing hypothyroidism. Thyrotropin-blocking autoantibodies (TBAb's) are typically polyclonal (10), and it is the general experience that they are of higher titer than TSAb's (for example, refs. 11, 12).

The TSHR antigen is unusual among the glycoprotein hormone receptors in undergoing intramolecular cleavage into a ligand-binding (TSH and autoantibody) A subunit and largely transmembrane B subunit (13, 14) with removal of an intervening $C$ peptide region $(15,16)$ (Figure 1a). This process also leads to shedding of heavily glycosylated A subunits from the cell surface (depicted in Figure 1c) $(17,18)$. It is noteworthy that there is no autoimmune response to the closely related gonadotropin hormone receptors that neither cleave nor shed portions of their ectodomains.

In experimental animals, there is no difficulty in generating Ab's to the TSHR by conventional immunization with purified antigen and adjuvant. However, unlike autoantibodies, most (reviewed in ref. 19), but not all (20), of these Ab's fail to activate the TSHR and do not produce hyperthyroidism. In contrast, TSAb's are much more effectively induced by injecting animals with cells 
coexpressing the TSHR and MHC class II molecules (20, 21) or following genetic immunization with plasmid (22) or with adenovirus (23) encoding the TSHR. Therefore, processing and presentation of TSHR expressed in vivo is likely to be essential to induce Ab's that recognize the TSHR epitope(s) necessary for receptor activation. Defining the TSHR epitopes involved in autoantibody activation is clearly an elemental goal in understanding how (and perhaps why) this remarkable phenomenon occurs and how the process may be aborted to provide a cure for Graves disease. TSAb's have conformational and discontinuous epitopes, a major component of which is at the $\mathrm{N}$-terminal region of the A subunit $(24,25)$ (Figure 1a), particularly involving a cysteine-rich segment $(9,26,27)$ with homology to an epidermal growth factor-like domain in laminin (28).

The present study provides novel and surprising information on the interaction between functional autoantibodies and the TSHR. TSAb's in Graves disease preferentially recognize the free A subunit and not the TSH holoreceptor. This observation supports the concept that shed A subunits either initiate or amplify the autoimmune response to the TSHR, thereby causing Graves disease.

\section{Methods}

Sera. Sera from 20 patients with hyperthyroid Graves disease were collected in the outpatient clinic or were kindly provided by Yuji Nagayama (Nagasaki University, Nagasaki, Japan). Sera from seven patients with hypothyroidism secondary to TBAb's were the gift of Luca Chiovato (University of Pisa, Pisa, Italy). The following murine mAb's were used: 3BD10 (epitope TSHR amino acid residues 25-51) (27) and 2C11 (epitope containing amino acid residues 354-359) (29).

Cell lines and culture. Generation of Chinese hamster ovary $(\mathrm{CHO})$ cells stably expressing human TSHR (TSHR-10,000 with an amplified transgenome) (30) and the TSHR ectodomain tethered to the plasma membrane by a glycosylphosphatidylinositol (ECDGPI) anchor (depicted in Figure 1b) (31) have been described previously. Cells were propagated in Ham's F-12 medium supplemented with $10 \%$ FCS, penicillin $(100 \mathrm{U} / \mathrm{ml})$, gentamicin $(50 \mu \mathrm{g} / \mathrm{ml})$, and amphotericin $\mathrm{B}(2.5 \mu \mathrm{g} / \mathrm{ml})$.

Flow cytometry. Intact $\mathrm{CHO}$ cells were harvested from 10-cm-diameter dishes using $1 \mathrm{mM}$ EDTA/1 mM EGTA in Dulbecco's PBS, pH 7.4. After washing twice with PBS containing $10 \mathrm{mM}$ HEPES, pH 7.4, 2\% heatinactivated FBS, and $0.05 \% \mathrm{NaN}_{3}$, the cells were incubated for 30 minutes at room temperature in $100 \mu \mathrm{l}$ of the same buffer containing $1 \mu \mathrm{g}$ of $\mathrm{mAb} 2 \mathrm{C} 11$ or $3 \mathrm{BD} 10$. As a negative control, cells were incubated in $100 \mu \mathrm{l}$ of normal mouse sera (1:100). Human sera were used at a dilution of 1:50. Where indicated in the text, incubations included TSHR-289 (100 ng/tube) purified as described previously (9). After rinsing, the cells were incubated for 1 hour at $4^{\circ} \mathrm{C}$ with $100 \mu$ l of FITCconjugated goat anti-mouse or anti-human $\operatorname{IgG}$
(1:100) (Caltag Laboratories Inc., Burlingame, California, USA), washed, and analyzed using a Beckman FACScan flow cytometer (Beckton Dickinson Immunocytometry Systems, San Jose, California, USA). Cells stained with propidium iodide $(1 \mu \mathrm{g} / \mathrm{ml}$ final concentration) were excluded from analysis.

Covalent cross-linking of radiolabeled TSH. Highly purified bovine TSH (National Hormone and Peptide Program, Torrance, California, USA) was radiolabeled with ${ }^{125}$ I to a specific activity of approximately $60 \mu \mathrm{Ci} / \mu \mathrm{g}$ using Bolton Hunter reagent $(4,400 \mathrm{Ci} / \mathrm{mmol}$; Perkin Elmer Life Sciences, Boston, Massachusetts, USA). Confluent 100-mmdiameter dishes of cells were incubated for 3 hours at $37^{\circ} \mathrm{C}$ with approximately $5 \mu \mathrm{Ci}{ }^{125} \mathrm{I}-\mathrm{TSH}$ followed by cross-linking with $1 \mathrm{mM}$ disuccinimidyl suberate (SigmaAldrich, St. Louis, Missouri, USA) and processed as
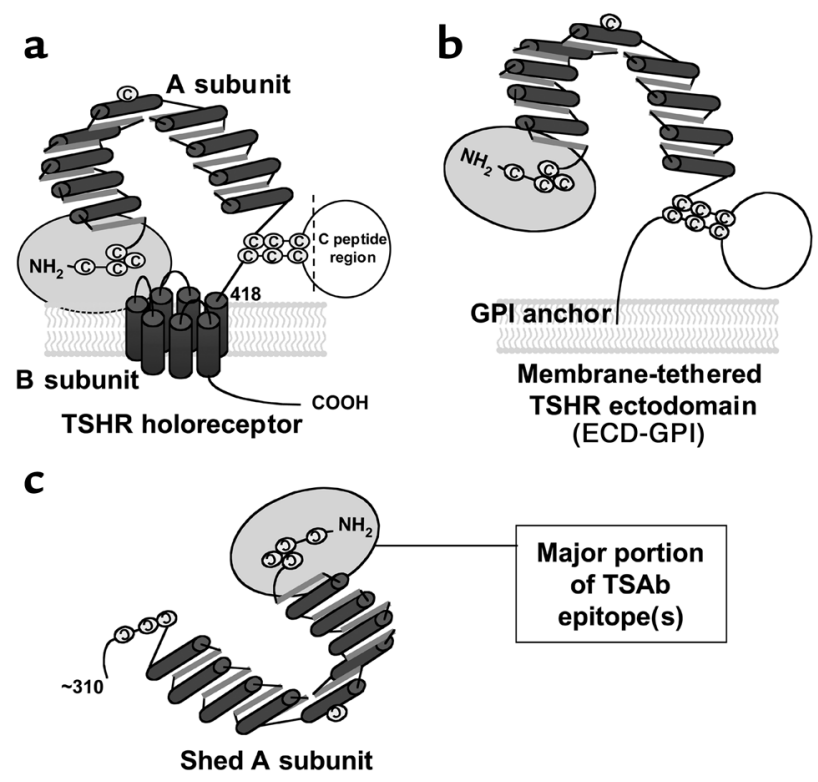

\section{Figure 1}

Schematic representation of different forms of the TSHR. (a) TSH holoreceptor. Intramolecular cleavage of the single polypeptide chain is followed by removal of the $C$ peptide region, with the $A$ subunit remaining tethered to the membrane-spanning $B$ subunit by disulfide bonds. The cylinders depict the $\alpha$ helices in the nine leucine-rich repeats in the A subunit, as well as the seven transmembrane segments of the $B$ subunit. The ectodomain comprises the entire A subunit and the extracellular region of the $B$ subunit. The ectodomain enters the plasma membrane at approximately amino acid residue 418 . A critical domain in the TSAb epitope(s) involves the cysteine-rich area at the extreme $\mathrm{N}$ terminus of the TSHR and is shown by the gray oval. (b) TSHR ectodomain tethered to the plasma membrane by a GPI anchor (ECD-GPI). This construct involved the attachment after TSHR codon 412 of a 39-amino acid sequence containing a signal for GPI attachment (31). The putative GPI attachment site of the anchor is at codon 425. Because of the additional length of the ectodomain prior to insertion into the membrane, and because the GPI anchor only traverses the outer leaf of the plasma membrane, the TSHR ectodomain is shown in a more "open" orientation relative to the wild-type TSHR. (c) Shed A subunit. The exact site of cleavage has not been established, but is approximately at amino acid residue 310 . The purified TSHR289 preparation used in this study is truncated at TSHR amino acid residue 289 and therefore comprises nearly the entire A subunit. 
described previously in detail (15). After addition of Laemmli sample buffer (32) containing $0.7 \mathrm{M} \beta$-mercaptoethanol $\left(30\right.$ minutes at $\left.50^{\circ} \mathrm{C}\right)$, the samples were electrophoresed on 10\% SDS-polyacrylamide gels (Bio-Rad Laboratories Inc., Hercules, California, USA). Prestained molecular-weight markers (Bio-Rad Laboratories Inc.) were included in parallel lanes. Radiolabeled proteins were visualized by autoradiography on Biomax MS x-ray film (Eastman Kodak Co., Rochester, New York, USA).

Immunoprecipitation of TSHR. The immunoprecipitation procedure used has been described previously in detail (30). Cells were pulsed $\left(1\right.$ hour at $\left.37^{\circ} \mathrm{C}\right)$ with 0.2 $\mathrm{mCi} / \mathrm{ml}$ of $\left.{ }^{[35} \mathrm{S}\right]$ methionine/cysteine in DMEM, high glucose $(4,500 \mathrm{mg} / \mathrm{l})$ methionine- and cysteine-free medium, containing $5 \%$ dialyzed FCS. After rinsing, cells were scraped into $1 \mathrm{ml}$ of ice-cold buffer A $(20 \mathrm{mM}$ HEPES, pH 7.2, $150 \mathrm{mM} \mathrm{NaCl}, 100 \mu \mathrm{g} / \mathrm{ml} \mathrm{PMSF}$, and 1 $\mu \mathrm{g} / \mathrm{ml}$ leupeptin; the latter two from Sigma-Aldrich). The cells were pelleted, rinsed, and resuspended in buffer A containing $1 \%$ Triton X-100. After 2 hours at $4^{\circ} \mathrm{C}$ with occasional vortexing, the mixture was centrifuged for 1 hour at $100,000 \mathrm{~g}$, and the supernatant was retained for immunoprecipitation. Solubilized cell proteins were diluted 1:1 in buffer B (20 mM HEPES, pH 7.2, $300 \mathrm{mM}$ $\mathrm{NaCl}, 0.1 \% \mathrm{SDS}, 0.5 \%$ Nonidet P-40, and the protease inhibitors described above) and were precleared with normal mouse serum IgG prebound to $25 \mu \mathrm{l}$ packed and washed protein A-agarose (Sigma-Aldrich). Mouse mAb A9 (1:1,000; kindly provided by Paul Banga, London, United Kingdom) was added (16 hours at $\left.4^{\circ} \mathrm{C}\right)$, and immune complexes were recovered using protein Aagarose (Sigma-Aldrich). After rinsing (five times with buffer $B$ and once with PBS), beads were resuspended in Laemmli sample buffer with $0.7 \mathrm{M} \beta$-mercaptoethanol $\left(30\right.$ minutes at $\left.50^{\circ} \mathrm{C}\right)$, aliquots of solubilized material were electrophoresed on $10 \%$ SDS-polyacrylamide gels (Bio-Rad Laboratories Inc.), and radiolabeled proteins were visualized by autoradiography as described above.

\section{Results}

Differential recognition of the TSHR ectodomain by murine TSHR $m A b$ 's. Recently, the TSHR ectodomain tethered to intact cells by a GPI anchor (ECD-GPI; Figure 1b) has been found to be very effective for detecting human autoantibody binding by flow cytometry $(12,33)$. The present concept for this observation is that the TSHR ECD-GPI is expressed approximately three to fivefold higher on the cell surface $(12,31)$ than the wild-type TSHR with seven membrane-spanning segments (for example 34-36). For this reason, we were puzzled by an observation that we made with murine $\mathrm{mAb}$ to the TSHR. The mAb 3BD10 has minimal recognition on flow cytometry of a cell line (TSHR-10,000) whose amplified transgenome leads to expression of an extremely high number $\left(2 \times 10^{6}\right)$ of TSHRs on the cell surface (30). To our surprise, 3BD10 recognized the ECD-GPI cells very well (Figure 2a).

Two possibilities existed to explain this apparent paradox. Either the level of expression was far lower in TSHR-10,000 cells than our previous data suggested (30), or mAb 3BD10 recognized the TSHR ectodomain with the GPI anchor far better than the ectodomain in the wild-type TSHR in the TSHR-10,000 cells. We therefore tested a different murine mAb, 2C11 (29), whose epitope is to another region of the TSHR ectodomain. In contrast to $3 \mathrm{BD} 10, \mathrm{mAb} 2 \mathrm{C} 11$ recognized TSHR-10,000 cells greater than tenfold better than the ECD-GPI cells (Figure $2 \mathrm{~b}$ ). This dramatic reversal in TSHR recognition is even greater in view of the fact that the 2C11 epitope (amino acid residues 354-359) (37) lies within a C peptide region deleted upon spontaneous TSHR intramolecular cleavage into $A$ and B subunits (reviewed in ref. 19). Therefore, $2 \mathrm{C} 11$ detects only residual, single polypeptide chain TSHRs on the cell surface. These data suggested that the level of TSHR expression was, indeed, far higher in TSHR10,000 than in ECD-GPI cells.
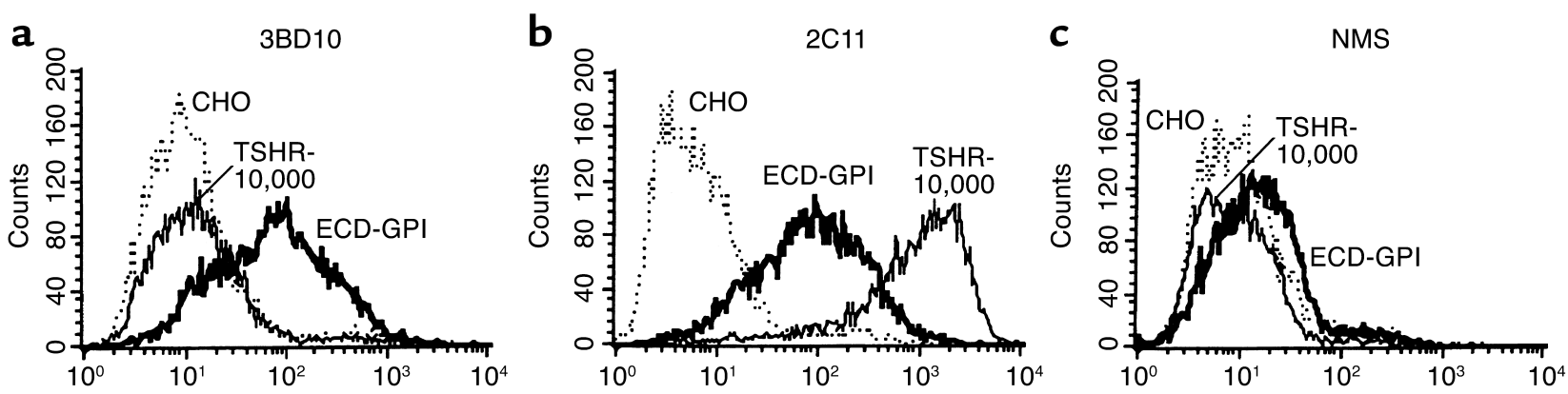

Figure 2

Differential recognition by murine TSHR mAb of the wild-type TSHR ectodomain and the GPI-anchored ectodomain. Flow cytometry was performed with the following murine mAb's: (a) mAb 3BD10, epitope including the cysteine-rich segment at the extreme $\mathrm{N}$ terminus of the TSHR ectodomain; (b) mAb 2C11, epitope in the C peptide region of the TSHR ectodomain, present only in the uncleaved, but not in the cleaved, TSHR; (c) normal mouse serum (NMS) included as a control. The assays used the following cell lines: TSHR-10,000, ECD-GPI, and $\mathrm{CHO}$. TSHR-10,000 is the $\mathrm{CHO}$ cell line stably expressing the wild-type TSH holoreceptor. The transgenome has been amplified using the dihydrofolate reductase minigene approach (adaptation to final methotrexate concentration of 10,000 nM), leading to very high TSHR expression on the cell surface $\left(\sim 2 \times 10^{6} /\right.$ cell $)(30)$. ECD-GPI is the $\mathrm{CHO}$ cell line stably expressing the TSHR ectodomain anchored to the plasma membrane by a GPI moiety. $\mathrm{CHO}$ are untransfected $\mathrm{CHO}$ cells. Note the approximately 100 -fold difference in 3BD10 versus $2 \mathrm{C} 11$ recognition of the TSHR holoreceptor, despite their similar recognition of the ECD-GPI cell line. 
a

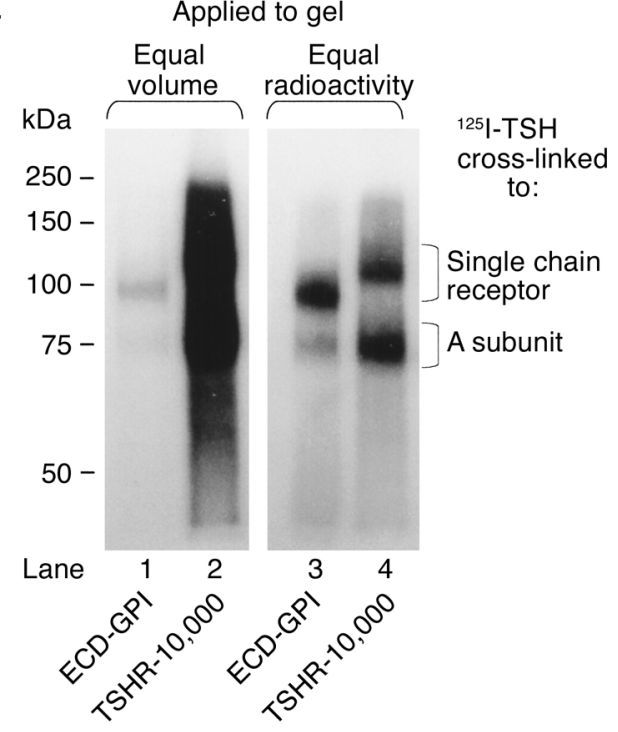

b

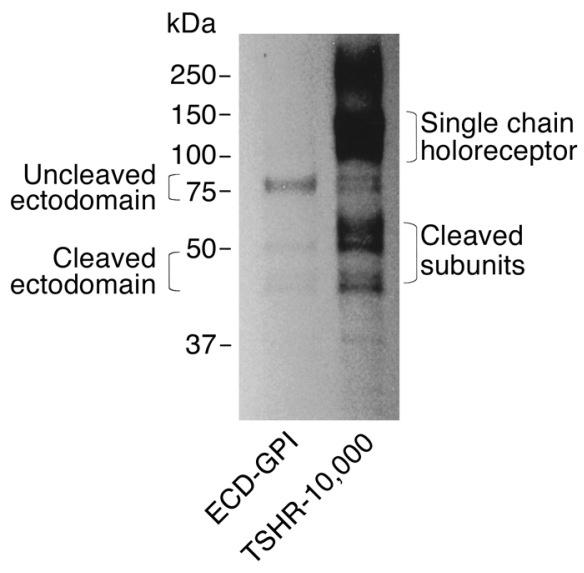

\section{Figure 3}

(a) TSH covalent cross-linking to intact cells reveals greater level of cell surface TSHR expression in TSHR-10,000 cells than in ECD-GPI cells. After simultaneous ${ }^{125}$ I-TSH cross-linking (same number of cells, same amount of ${ }^{125}$ I-TSH), cell extracts were subjected to polyacrylamide gel electrophoresis (10\%) under reducing conditions (see Methods). Autoradiography (representative of three experiments) was for 16 hours. In lanes 1 and 2, equal volumes of cell extract were applied. Lanes 3 and 4 were loaded with equal amounts of radioactivity. TSH cross-linking to intact cells, unlike immunoprecipitation (b), visualizes only mature TSHR expressed on the cell surface. Two forms of TSHR are seen: single-chain uncleaved TSHR and the ligand binding A subunit in the cleaved TSHR released from the serpentine region (TSHR-10,000 cells) or from the GPI-anchored portion (ECD-GPI) by disulfide bond reduction. Note the lesser proportion of cleaved versus uncleaved receptors in the ectodomain with the GPI anchor than in the wild-type TSHR. (b) Immunoprecipitation confirms higher level of TSHR expression in TSHR-10,000 cells than in ECD-GPI cells. Similar numbers of TSHR-10,000 cells and ECD-GPI cells were precursor labeled simultaneously with the same quantity of ${ }^{35} \mathrm{~S}$-methionine and ${ }^{35} \mathrm{~S}$-cysteine (see Methods). Detergent-solubilized particulate preparations were immunoprecipitated with murine mAb A9 (epitope in the midregion of the TSHR ectodomain), the most effective mAb available to us for immunoprecipitating all forms of the TSHR. After polyacrylamide gel electrophoresis (10\%), autoradiography (representative of duplicate experiments) was performed for 16 hours.

The potential pathophysiological importance of the differential wild-type TSHR/ECD-GPI recognition was that the 3BD10 epitope includes the cysteine cluster at the N-terminus of the TSHR ectodomain, a critical region for TSAb action. It was, therefore, important to confirm by methods other than flow cytometry that the TSHR-10,000 cells did, indeed, express more receptors on the cell surface than the ECD-GPI cells. For this purpose, we cross-linked ${ }^{125}$ I-TSH to the surface of intact cells in a monolayer. This approach detects only ligandbinding forms of the TSHR on the cell surface and provides a clear profile of TSHR subunit composition. When equal volumes of material were applied to the gel after TSH cross-linking, the autoradiographic exposure required to obtain a detectable signal with the ECD-GPI cells (Figure 3a, lane 1) created an autoradiographic signal so strong with the TSHR-10,000 cells that we were unable to discern the cleaved and uncleaved forms of the TSHR (Figure 3a, lane 2). Normalization of the amount of radioactivity applied to the gel did allow visualization of the subunit composition of the two different forms of TSHR. As reported previously (reviewed in ref. 19), a proportion of ligand-binding wild-type TSHR on the cell surface had cleaved into two subunits, with the ligand-binding A subunit released by disulfide bond reduction from the membrane-spanning B subunit (Figure 3a, lane 4). In contrast to the wild-type TSHR, most of the ECD-GPI ectodomain remained uncleaved (Figure 3a, lane 3). Incidentally, the very high number of receptors on TSHR-10,000 cells precludes determination of receptor number by Scatchard analysis of TSH-binding kinetics (30).

Further confirmation of greater TSHR expression in the TSHR-10,000 versus ECD-GPI cells was obtained by receptor immunoprecipitation in detergent-solubilized precursor-labeled cells. For this purpose we used another $\mathrm{mAb}$ (A9) with an epitope in the midregion of the TSHR ectodomain (38). Immunoprecipitation provides qualitatively more complex information on the TSHR (cell surface and intracellular, synthetic and degradation pathways). Multiple bands were observed on autoradiography, consistent with previous reports on the ECD-GPI (31) and various forms of the wild-type TSHR, including its subunits (19) (Figure 3b). Most important for the present study, the quantity of TSHR immunoprecipitated was far greater from the TSHR-10,000 than from the ECD-GPI lysate.

Based on the TSH cross-linking and immunoprecipitation studies, the level of TSHR expression was clearly 

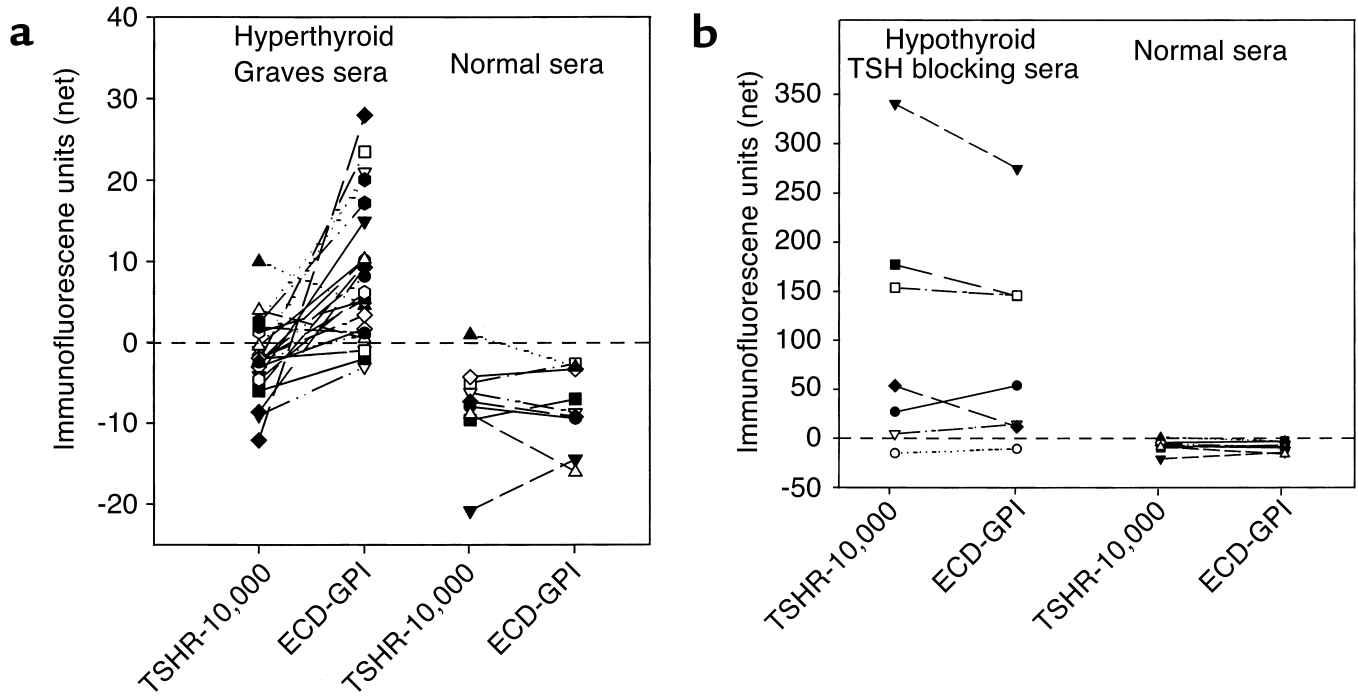

\section{Figure 4}

(a) Stimulating TSHR autoantibodies recognize the wild-type TSHR less well than the TSHR ectodomain with a GPI anchor. Sera (diluted 1:50) from hyperthyroid Graves patients, all with high levels of TSHR autoantibodies, were tested by flow cytometry on cells expressing the wild-type TSH holoreceptor (TSHR-10,000) and the TSHR ectodomain with a GPI anchor (see Methods). Net mean fluorescence values for the Graves sera (and for control sera from normal individuals) are plotted after subtraction of background fluorescence obtained with untransfected $\mathrm{CHO}$ cells. Statistical significance of differences between individual Graves serum recognition of the TSHR-10,000 and the ECD-GPI cells was determined by the paired $t$ test; $P<0.001$. (b) Sera from rare hypothyroid patients with TBAb's show no difference in their recognition of the wild-type TSHR and the GPI-anchored TSHR ectodomain. Sera (diluted 1:50) were tested by flow cytometry on cells expressing the wild-type TSH holoreceptor (TSHR-10,000) and the TSHR ectodomain with a GPI anchor (see Methods). Net mean fluorescence values for the sera (and for control sera from normal individuals) are indicated after subtraction of background fluorescence obtained with untransfected $\mathrm{CHO}$ cells. Note the difference scales for the $\mathrm{Y}$ axes in $\mathbf{a}$ and $\mathbf{b}$, reflecting the higher titers of TBAb's versus TSAb's.

higher in the TSHR-10,000 cells than in the ECD-GPI cells. Therefore, preferential recognition by mAb 3BD10 of the ECD-GPI cells could not be attributed to greater expression of this form of the TSHR relative to the wildtype TSHR in the TSHR-10,000 cells. A final consideration was that the high receptor density on the TSHR10,000 cells could be responsible for poor recognition by $\mathrm{mAb} 3 \mathrm{BD} 10$, perhaps by receptor clustering leading to steric hindrance. However, this possibility could be excluded because the same differential recognition between $\mathrm{mAb} 3 \mathrm{BD} 10$ and $\mathrm{mAb} 2 \mathrm{C} 11$ with the TSHR10,000 cells (Figure 2 ) was observed with a cell line (TSHR-0) without an amplified transgenome expressing only approximately 150,000 TSHR per cell (30). Thus, flow cytometry with TSHR-0 cells yielded fluorescence values of 96.47 for $2 \mathrm{C} 11$ and 11.55 for 3BD10 (untransfected cells 7.50 and 14.86, respectively).

Differential recognition of the TSHR ectodomain by buman TSHR autoantibodies. The remarkable discordance between murine $\mathrm{mAb}$ in their recognition of the wild- a

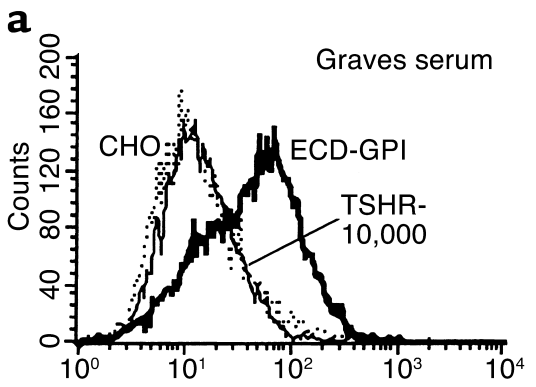

b

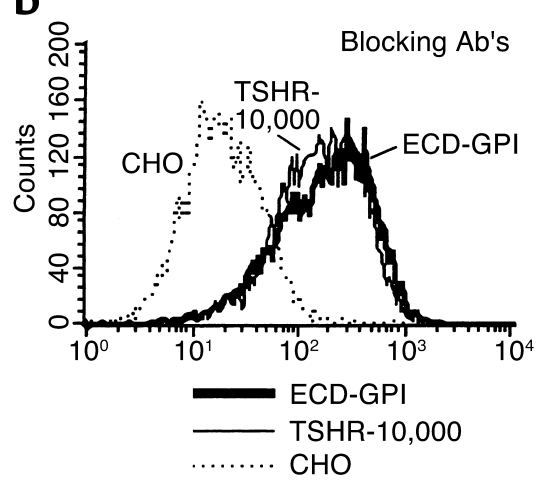

c

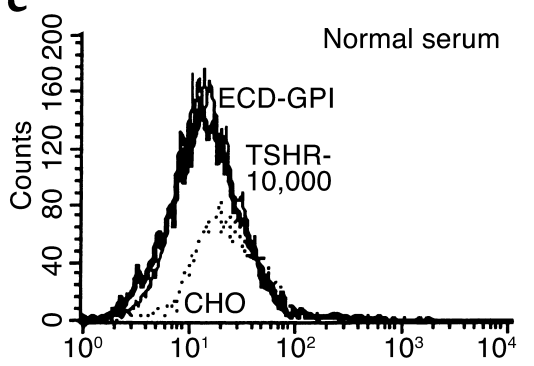

Figure 5

Examples of flow cytometry data obtained with a Graves hyperthyroid serum and serum from a hypothyroid patient with TBAb activity. Note that despite giving an excellent fluorescent signal on the ECD-GPI cell line, the same Graves serum does not recognize the TSHR-10,000 cells overexpressing the TSH holoreceptor on their surface (a). In contrast, the serum from a hypothyroid patient with TBAb activity recognizes both ECD-GPI and TSHR-10,000 cells equally well (b). Serum from a normal individual is included as a control (c). 


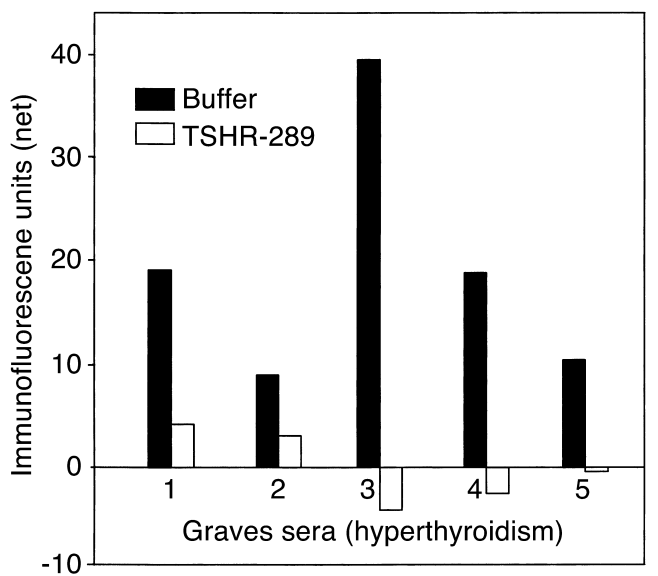

Figure 6

Neutralization by purified TSHR A subunits of TSHR autoantibodies from Graves patients with hyperthyroidism. Flow cytometry was performed using sera from five Graves patients whose signal upon flow cytometry gave the largest differential between recognition of the wild-type TSHR and GPI-anchored ectodomain (Figure 4a). Sera were preincubated in either buffer alone or in buffer containing a large excess of TSHR-289 $(0.1 \mu \mathrm{g} / 0.1 \mathrm{ml}$ of serum diluted $1: 50)$ before addition to $\mathrm{CHO}$ cells expressing ECD-GPI. TSHR-289 is a secreted, soluble, heavily glycosylated protein that comprises amino acid residues 22-289 of the TSHR, essentially the A subunit (residues 1-21 being the signal peptide). Net fluorescence values for the sera are indicated after subtraction of background fluorescence obtained with untransfected $\mathrm{CHO}$ cells.

type TSH holoreceptor and the GPI-anchored TSHR ectodomain led us to examine by flow cytometry the relative recognition of these two ectodomain forms by TSAb's and TBAb's. We studied sera from hyperthyroid Graves patients, all with high levels of TSHR autoantibodies detected by a standard clinical assay (TSH-binding inhibition; values ranging from $40-92 \%$ inhibition). Remarkably, 17 of 20 sera recognized the ECD-GPI cells better than the TSHR-10,000 cells (Figure 4a; $P<0.001$, paired $t$ test) despite the greater number of TSHR expressed on the TSHR-10,000 cells. There was no recognition of either cell type by nine sera from normal individuals. There was a significant correlation between TSAb activity (range of $220-2,000 \%$ of the basal cAMP values) and the flow cytometry data obtained with the ECD-GPI cells $(r=0.645 ; P<0.02)$.

\section{Figure 7}

Variable neutralization by purified TSHR A subunits of TSHR autoantibodies from hypothyroid patients with TBAb's. Flow cytometry was performed using sera from seven patients. Sera were preincubated in either buffer alone or in buffer containing TSHR-289 $(0.1 \mu \mathrm{g} / 0.1 \mathrm{ml}$ of serum diluted 1:50) before addition to $\mathrm{CHO}$ cells expressing the wild-type (TSHR-10,000 cells) or the GPI-anchored TSHR ectodomain (ECD-GPI cells). Net fluorescence values for the sera are indicated after subtraction of background fluorescence obtained with untransfected $\mathrm{CHO}$ cells. Note the difference scales for the $Y$ axes here and in Figure 6, reflecting the higher titers of TBAb versus TSAb.
Very rarely, TSHR autoantibodies without agonist activity inhibit TSH binding, thereby causing hypothyroidism. Seven sera from hypothyroid patients with TBAb's were similarly tested on the two TSHR cell lines (Figure 4b). Unlike the Graves sera, the sera with TSHblocking activity from hypothyroid patients did not recognize the ECD-GPI cells better than the TSHR10,000 cells. Again, as controls, sera from normal individuals recognized neither form of the TSHR. Moreover, the majority of these sera gave much stronger signals on flow cytometry than did the sera from the hyperthyroid Graves patients (note the different scales for the ordinates on Figure $4, b$ vs. a, reflecting the higher titers of TBAb vs. TSAb). Examples of flow cytometry data obtained with a Graves hyperthyroid serum and serum from a hypothyroid patient with TBAb activity are shown in Figure 5.

Neutralization of TSHR autoantibody binding by purified TSHR A subunit. The question arises as to why TSHR autoantibodies in the sera of patients with Graves hyperthyroidism recognize the GPI-anchored ectodomain better than the wild-type TSHR. The autoantibody-binding ectodomains in both forms of receptor are identical, the only difference being their attachment to the plasma membrane. One possible explanation for the phenomenon was greater steric hindrance for autoantibody binding to the wild-type TSHR relative to the ECD-GPI. If correct, autoantibody binding should be neutralized by purified untethered A subunits in solution.

We tested sera from five Graves patients whose signal on flow cytometry gave the largest differential between

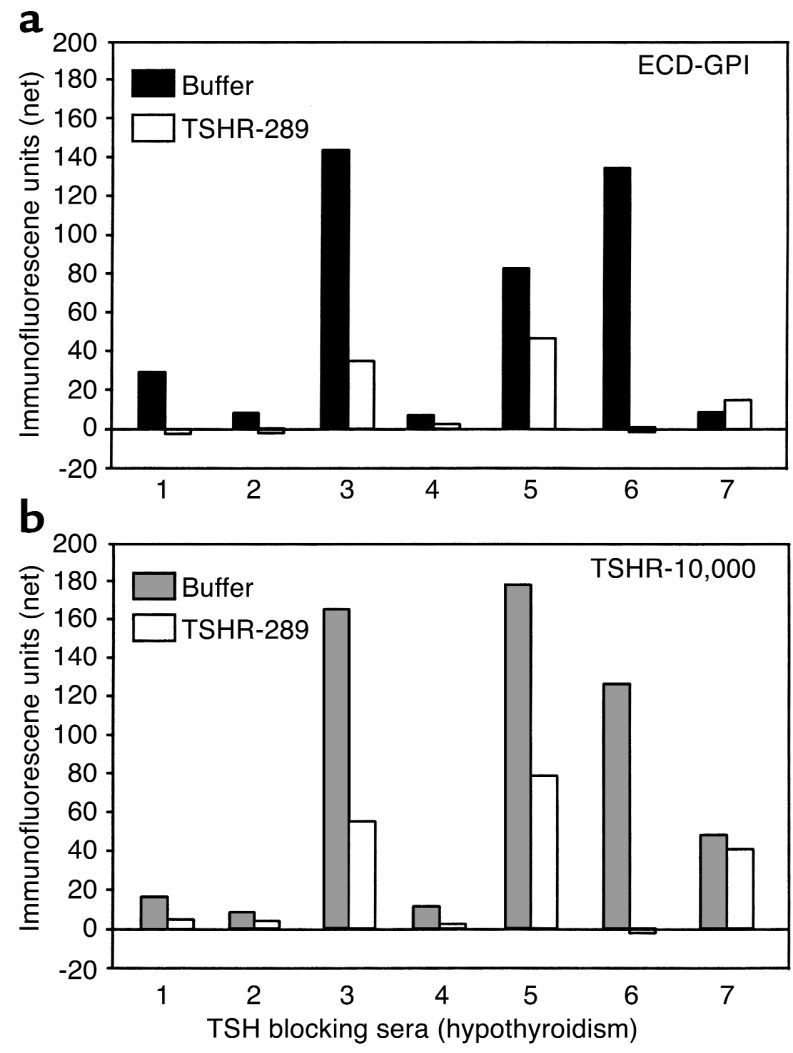


recognition of the wild-type TSHR and GPI-anchored ectodomain. TSHR-289 is a secreted, soluble, heavily glycosylated protein that comprises amino acid residues 22-289 of the TSHR, essentially the A subunit (residues 1-21 being the signal peptide). Sera were preincubated with purified TSHR-289 before addition to $\mathrm{CHO}$ cells expressing ECD-GPI. Note that the very low signals provided by the Graves sera with the wild-type TSHR (Figure 4a) precluded absorption studies with the TSHR10,000 cells. In all five sera, TSHR autoantibody binding was completely (or nearly completely in one serum) neutralized by soluble TSHR-289 (Figure 6).

In addition, we tested neutralization by TSHR-289 of the seven sera from rare patients with TBAb as the basis for their hypothyroidism. Unlike the hyperthyroid Graves sera, the high fluorescence values of these "blocking" sera permitted study with both the wild-type TSHR and the GPI-anchored ectodomain (Figure 4b). Again, unlike the Graves hyperthyroidism sera, some but not all of the TBAb sera were fully, or nearly fully, neutralized by TSHR 289. A wide range of neutralizing efficacy was observed, with similar degrees of neutralization being obtained with the ECD-GPI cells (Figure 7a) and the TSHR-10,000 cells (Figure 7b). Moreover, the extent of neutralization of TSHR autoantibody binding by TSHR289 was not a function of autoantibody concentration. No neutralization occurred with serum number 7 , which had low Ab binding to both cell types, whereas serum number 6 was totally neutralized despite being a more potent autoantibody. These data indicate that contrary to TSAb sera, the epitopes for TBAb are more variable and may, or may not, be present in the TSHR A subunit.

\section{Discussion}

A fortuitous observation has led to fundamental and important new insight with respect to TSHR autoantibodies in the pathogenesis of Graves disease. This observation was the differential recognition by two murine mAb's of the TSHR ectodomain when part of the holoreceptor on the cell surface or when attached to the cell membrane by a GPI anchor. We found that $\mathrm{mAb} 2 \mathrm{C} 11$ provided a signal more than 100 -fold greater than mAb 3BD10 with the wild-type TSHR, whereas both mAb's generated similar signals on the TSHR ECD-GPI. The relevance of this finding was that the 3BD10 epitope contains the cluster of cysteine residues at the $\mathrm{N}$ terminus of the TSHR ectodomain (27), shown to be critical in the action of TSAb's in Graves disease (28). Indeed, as with 3BD10, we subsequently found that the epitope(s) for TSAb's in Graves patients is largely obscured in the wild-type TSHR, but not in the GPI-anchored TSHR ectodomain.

This phenomenon explains, at least in part, the disagreement in the literature on the efficacy of detecting TSHR autoantibodies by flow cytometry using cells stably expressing the TSHR. Detection of TSHR autoantibodies by this means has not been straightforward, particularly because of their very low concentration (nanogram per milliliter range) in Graves patient serum (6-9). In our expe- rience, using cells expressing the wild-type TSHR, only a few Graves sera, those with the highest titer of TSHR autoantibodies provided a clear positive signal upon flow cytometry (7). In contrast, cells expressing the GPIanchored ectodomain have been much more effective (12, 33). These ECD-GPI cell lines express more receptors than other cell lines expressing the wild-type TSHR. However, in the present study we took advantage of a cell line with an amplified transgenome that we show expresses far more wild-type TSHR than the ECD-GPI cell line. Our data clearly establish that preferential recognition by Graves sera of the ECD-GPI is not related to the level of expression, but rather to the accessibility of the TSAb epitope. At face value, this finding could question the validity of some of the previous evidence for the low concentration of TSAb's in Graves serum. However, quantitative neutralization studies of TSHR autoantibodies using purified, recombinant TSHR A subunits substantiates the concept of very low TSAb concentrations in serum $(8,9)$. Also of interest, the phenomenon that we describe may explain the experience of Costagliola et al., who were unable to obtain thyroid-stimulating $\mathrm{mAb}$ from mice made hyperthyroid by TSHR-DNA vaccination (39). Screening for TSHR mAb was performed on cells expressing the wild-type TSHR, which we find is not recognized by sera from patients with TSAb activity.

An important question is why the wild-type TSHR on the cell surface is less well recognized than the ECD-GPI by $\mathrm{mAb} 3 \mathrm{BD} 10$ and by TSAb's. Murine mAb 3BD10 has the remarkable property of recognizing only one of two different conformational forms of the A subunit "module," TSHR-289 $(9,27)$. TSHR autoantibodies recognize the other conformational form of TSHR-289. This reciprocally exclusive recognition by $\mathrm{mAb} 3 \mathrm{BD} 10$ and autoantibodies involves the same cysteine-rich epitopic region at the extreme $\mathrm{N}$ terminus of the TSHR ectodomain. The possibility then arises that the ectodomain on the wildtype TSHR, unlike the ECD-GPI, is largely in the autoantibody "active" form and therefore is not recognized by 3BD10. However, this possibility is excluded because TSAb's, which recognize the active form, also poorly recognize the wild-type TSHR upon flow cytometry.

The only difference between the TSHR ectodomains on the two cell types is in their attachment to the plasma membrane. Furthermore, consistent with previous observations of a small, soluble protein released by freeze-thawing of thyroid tissue $(40,41)$, purified, untethered A subunits in solution effectively neutralize the binding of Graves TSHR autoantibodies to the receptor expressed on the cell surface. Taken together, this information provides strong evidence that the explanation for the discrepancy described above is greater steric hindrance to TSAb binding to the wildtype versus the GPI-anchored TSHR ectodomain. Four possible explanations for this disparity are steric hindrance by the plasma membrane, steric hindrance by the extracellular loops in the serpentine region of the TSH holoreceptor, steric hindrance upon multimerization of the wild-type TSHR with no (or lesser) mul- 
timerization of the ECD-GPI, and greater flexibility in that the GPI anchor increases TSAb epitope exposure. Regardless of which explanation is correct, all involve steric hindrance for TSAb binding.

Steric hindrance involving TSAb binding to the TSHR ectodomain raises an apparent paradox. If TSAb's have difficulty binding to the wild-type TSHR, how can they activate the receptor and cause hyperthyroidism? Obviously, TSAb binding to the TSH holoreceptor must occur, even if diminished. We can suggest two possible explanations for this phenomenon. First, very few TSHR (whether monomer or multimer) have to be engaged by TSAb to produce the clinical syndrome of hyperthyroidism. Indeed, marginally elevated serum TSH levels are sufficient to cause goiter in areas of mild iodine deficiency. Partial obstruction of TSAb binding to its epitope(s) may also explain the mechanism by which these autoantibodies can activate the TSHR. The binding interaction between an $\mathrm{Ab}$ and its binding site is remarkably flexible, or "plastic" (42). Such plasticity may be more marked when an epitope is partially obstructed and could thereby have a torsional effect on the TSHR ectodomain. Alternatively, partial obstruction of TSAb binding may involve a direct mechanical effect of the Ab itself on the TSHR ectodomain or extracellular loops.

A second possible explanation for the apparent paradox is that TSHR multimerization (43) is only partial, and monomeric forms of the TSHR remain accessible to TSAb. Of interest, TSH-binding affinity for the TSH holoreceptor is inversely proportional to the level of receptor expression (30). Such "negative cooperativity" must involve TSHR interactions. Therefore, TSHR overexpression may increase receptor multimerization and reduce autoantibody recognition. Against this hypothesis, however, is that upon flow cytometry, autoantibodies in Graves sera recognize the wild-type TSHR poorly or not at all, even when few receptors are expressed on the cell surface (7).

Our study provides interesting insight into differences between stimulating TSHR autoantibodies (TSAb's) and TBAb's that lack agonist activity and cause hypothyroidism. There is considerable confusion in the literature resulting from studies that measure TSH-blocking activity in sera with weak agonist (TSAb) activity assay (44). True TSHR-blocking Ab's lack agonist activity and cause hypothyroidism. Such patients are very rare. Titers in the TBAb sera that we studied were generally higher than those in the Graves hyperthyroid sera. In addition, purified TSHR A subunits (TSHR-289) neutralized TSHRbinding activity in the majority (but not all) of TBAb sera, in a manner unrelated to autoantibody titer. These data do not support the prevailing concept that the epitope(s) for blocking autoantibodies are largely at the $\mathrm{C}$ terminus of the TSHR ectodomain. Rather, they substantiate preliminary evidence for greater epitopic diversity among TBAb than TSAb sera (44).

Finally, the most important deduction from our data, in our view, relates to the origin of stimulatory TSHR autoantibodies that directly cause Graves disease. Although monoclonal human TSHR autoantibodies have yet to be obtained (45), analysis of the genes of human thyroid peroxidase autoantibodies indicate that thyroid autoimmunity is an antigen-driven process. The question then arises, what form of the TSHR is driving affinity maturation of TSAb-specific B cells? Our data clearly indicate preferential recognition by TSAb of the TSHR ectodomain (unencumbered by the plasma membrane or extracellular loops) and not the TSH holoreceptor. Only the shed A subunit can provide this antigen-driven affinity maturation. This deduction is supported by effective TSHR A subunit neutralization of TSAb activity, the antigenic immunodominance of the extreme $\mathrm{N}$ terminus of the A subunit when mice are immunized with purified A subunit (27), the induction of primarily TSH blocking Ab's on immunizing mice with the TSH holoreceptor (22), and the absence of a "Graves' disease of the gonads." Despite the high homology between cleaving and nonshedding TSHR and noncleaving and shedding gonadotropin receptors, stimulatory autoantibodies arise only against the former.

In conclusion, we provide evidence that TSAb's in Graves disease preferentially recognize the free A subunit and not the TSH holoreceptor. This observation supports the concept that shed A subunits either initiate or amplify the autoimmune response to the TSHR, thereby causing Graves disease.

\section{Acknowledgments}

This work was supported by NIH grants DK-19289 and DK-54684, as well as generous contributions from Boris Catz. We thank Yuji Nagayama and Luca Chiovato for kindly providing us with some of the sera used in the study.

1. Rapoport, B., and McLachlan, S.M. 2001. Thyroid autoimmunity. J. Clin. Invest. 108:1253-1259. doi:10.1172/JCI200114321.

2. Zakarija, M. 1983. Immunochemical characterization of the thyroidstimulating antibody (TSAb) of Graves' disease: evidence for restricted heterogeneity. J. Clin. Lab. Immunol. 10:77-85.

3. Knight, J., Laing, P., Knight, A., Adams, D., and Ling, N. 1986. Thyroid stimulating autoantibodies usually contain only lambda-light chains: evidence for the "forbidden clone" theory. J. Clin. Endocrinol. Metab. 62:342-347.

4. Williams, R.C., Jr., et al. 1988. Kappa/lambda immunoglobulin distribution in Graves' thyroid-stimulating antibodies. Simultaneous analysis of C lambda gene polymorphisms. J. Clin. Invest. 82:1306-1312.

5. Weetman, A.P., et al. 1990. Thyroid-stimulating antibody activity between different immunoglobulin G subclasses. J. Clin. Invest. 86:723-727.

6. De Forteza, R., Smith, C.U., Amin, J., McKenzie, J.M., and Zakarija, M. 1994. Visualization of the thyrotropin receptor on the cell surface by potent autoantibodies. J. Clin. Endocrinol. Metab. 78:1271-1273.

7. Jaume, J.C., Kakinuma, A., Chazenbalk, G.D., Rapoport, B., and McLachlan, S.M. 1997. TSH receptor autoantibodies in serum are present at much lower concentrations than thyroid peroxidase autoantibodies: analysis by flow cytometry. J. Clin. Endocrinol. Metab. 82:500-507.

8. Chazenbalk, G.D., Jaume, J.C., McLachlan, S.M., and Rapoport, B. 1997. Engineering the human thyrotropin receptor ectodomain from a nonsecreted form to a secreted, highly immunoreactive glycoprotein that neutralizes autoantibodies in Graves' patients' sera. J. Biol. Chem. 272:18959-18965.

9. Chazenbalk, G., McLachlan, S., Pichurin, P., and Rapoport, B. 2001. A "prion-like" shift between two conformational forms of a recombinant thyrotropin receptor A subunit module: purification and stabilization 
using chemical chaperones of the form reactive with Graves' autoantibodies. J. Clin. Endocrinol. Metab. 86:1287-1293.

10. Kraiem, Z., et al. 1992. The IgG subclass distribution of TSH receptor blocking antibodies in primary hypothyroidism. Clin. Endocrinol. 37:135-140.

11. Maruyama, M., et al. 2001. Reactivity of thyrotropin receptor autoantibodies with the thyrotropin receptor on Western blots. Thyroid. 11:211-219.

12. Costagliola, S., Khoo, D., and Vassart, G. 1998. Production of bioactive amino-terminal domain of the thyrotropin receptor via insertion in the plasma membrane by a glycosylphosphatidylinositol anchor. FEBS Lett. 436:427-433.

13. Buckland, P.R., Rickards, C.R., Howells, R.D., Jones, E.D., and Rees Smith, B. 1982. Photo-affinity labeling of the thyrotropin receptor. FEBS Lett. 145:245-249.

14. Loosfelt, H., et al. 1992. Two-subunit structure of the human thyrotropin receptor. Proc. Natl. Acad. Sci. USA. 89:3765-3769.

15. Chazenbalk, G.D., et al. 1997. Evidence that the thyrotropin receptor ectodomain contains not one, but two, cleavage sites. Endocrinology. 138:2893-2899.

16. de Bernard, S., et al. 1999. Sequential cleavage and excision of a segment of the thyrotropin receptor ectodomain. J. Biol. Chem. 274:101-107.

17. Couet, J., et al. 1996. Shedding of human thyrotropin receptor ectodomain: involvement of a matrix metalloprotease. J. Biol. Chem. 271:4545-4552.

18. Tanaka, K., Chazenbalk, G.D., McLachlan, S.M., and Rapoport, B. 1999 The shed component of the TSH receptor is primarily a carboxyl terminal truncated form of the A subunit, not the entire A subunit. Molec. Cell. Endocrinol. 150:113-119.

19. Rapoport, B., Chazenbalk, G.D., Jaume, J.C., and McLachlan, S.M. 1998. The thyrotropin receptor: interaction with thyrotropin and autoantibodies. Endocr. Rev. 19:673-716.

20. Kaithamana, S., Fan, J., Osuga, Y., Liang, S.G., and Prabhakar, B.S. 1999. Induction of experimental autoimmune Graves' disease in BALB/c mice. J. Immunol. 163:5157-5164.

21. Shimojo, N., et al. 1996. Induction of Graves-like disease in mice by immunization with fibroblasts transfected with the thyrotropin receptor and a class II molecule. Proc. Natl. Acad. Sci. USA. 93:11074-11079.

22. Costagliola, S., et al. 2000. Genetic immunization of outbred mice with thyrotropin receptor cDNA provides a model of Graves' disease. J. Clin. Invest. 105:803-811.

23. Nagayama, Y., et al. 2002. A novel murine model of Graves' hyperthyroidism with intramuscular injection of adenovirus expressing the thyrotropin receptor. J. Immunol. 168:2789-2794.

24. Nagayama, Y., Wadsworth, H.L., Russo, D., Chazenbalk, G.D., and Rapoport, B. 1991. Binding domains of stimulatory and inhibitory thyrotropin (TSH) receptor autoantibodies determined with chimeric TSHlutropin/chorionic gonadotropin receptors. J. Clin. Invest. 88:336-340.

25. Tahara, K., Ban, T., Minegishi, T., and Kohn, L.D. 1991. Immunoglobulins from Graves' disease patients interact with different sites on TSH receptor/LH-CG receptor chimeras than either TSH or immunoglobulins from idiopathic myxedema patients. Biochem. Biophys. Res. Comm. 179:70-77.

26. Nagayama, Y., and Rapoport, B. 1992. Thyroid stimulatory autoantibodies in different patients with autoimmune thyroid disease do not all recognize the same components of the human thyrotropin receptor: selective role of receptor amino acids Ser25-Glu30. J. Clin. Endocrinol. Metab. 75:1425-1430.

27. Chazenbalk, G.D., et al. 1999. A mouse monoclonal antibody to a thyrotropin receptor ectodomain variant provides insight into the exquisite antigenic conformational requirement, epitopes and in vivo concentra- tion of human autoantibodies. J. Clin. Endocrinol. Metab. 84:702-710.

28. Chen, C.-R., Tanaka, K., Chazenbalk, G.D., McLachlan, S.M., and Rapoport, B. 2001. A full biological response to autoantibodies in Graves' disease requires a disulfide-bond loop in the thyrotropin N-terminus homologous to a laminin EGF-like domain. J. Biol. Chem. 276:14767-14772.

29. Johnstone, A.P., Cridland, J.C., DaCosta, C.R., Harfst, E., and Shepherd, P.S. 1994. Monoclonal antibodies that recognize the native human thyrotropin receptor. Mol. Cell. Endocrinol. 105:R1-R9.

30. Chazenbalk, G.D., Kakinuma, A., Jaume, J.C., McLachlan, S.M., and Rapoport, B. 1996. Evidence for negative cooperativity among human thyrotropin receptors overexpressed in mammalian cells. Endocrinology. 137:4586-4591.

31. Da Costa, C.R., and Johnstone, A.P. 1998. Production of the thyrotropin receptor extracellular domain as a glycosylphosphatidylinositolanchored membrane protein and its interaction with thyrotropin and autoantibodies. J. Biol. Chem. 273:11874-11880.

32. Laemmli, U.K. 1970. Cleavage of structural proteins during the assembly of the head of bacteriophage T4. Nature. 227:680-685.

33. Metcalfe, R., et al. 2002. Demonstration of immunoglobulin G, A, and E autoantibodies to the human thyrotropin receptor using flow cytometry. J. Clin. Endocrinol. Metab. 87:1754-1761.

34. Harfst, E., and Johnstone, A.P. 1992. Characterization of the glutamine synthetase amplifiable eukaryotic expression system applied to an integral membrane protein - the human thyrotropin receptor. Anal. Biochem. 207:80-84.

35. Costagliola, S., et al. 1992. Binding assay for thyrotropin receptor autoantibodies using the recombinant receptor protein. J. Clin. Endocrinol. Metab. 75:1540-1544

36. Kakinuma, A., Chazenbalk, G., Filetti, S., McLachlan, S.M., and Rapoport, B. 1996. Both the $5^{\prime}$ and $3^{\prime}$ non-coding regions of the thyrotropin receptor messenger RNA influence the level of receptor protein expression in transfected mammalian cells. Endocrinology. 137:2664-2669.

37. Van Sande, J., et al. 1996. Specific activation of the thyrotropin receptor by trypsin. Mol. Cell. Endocrinol. 119:161-168.

38. Nicholson, L.B., et al. 1996. Monoclonal antibodies to the human thyrotropin receptor: epitope mapping and binding to the native receptor on the basolateral plasma membrane of thyroid follicular cells. J. Mol. Endocrinol. 16:159-170.

39. Costagliola, S., Rodien, P., Many, M.-C., Ludgate, M., and Vassart, G. 1998. Genetic immunization against the human thyrotropin recepto causes thyroiditis and allows production of monoclonal antibodies recognizing the native receptor. J. Immunol. 160:1458-1465.

40. Smith, B.R. 1970. The interaction of the long-acting thyroid stimulator (LATS) with thyroid tissue in vitro. J. Endocrinol. 46:45-54.

41. Davies Jones, E., and Rees Smith, B. 1984. A water-soluble fragment of the thyroid-stimulating hormone receptor which binds both thyroidstimulating hormone and thyroid-stimulating hormone receptor antibodies. J. Endocrinol. 100:113-118.

42. Nair, D.T., et al. 2002. Epitope recognition by diverse antibodies suggests conformational convergence in an antibody response. J. Immunol. 168:2371-2382

43. Latif, R., Graves, P., and Davies, T.F. 2001. Oligomerization of the human thyrotropin receptor. Fluorescent protein-tagged hRSHR reveals posttranslational complexes. J. Biol. Chem. 276:45217-45224.

44. Schwarz-Lauer, L., et al. 2002. Evidence for a simplified view of autoantibody interactions with the TSH receptor. Thyroid. 12:115-120.

45. McLachlan, S.M., and Rapoport, B. 1996. Monoclonal human autoantibodies to the TSH receptor: the holy grail, and why are we looking for it? J. Clin. Endocrinol. Metab. 81:3152-3154 